\title{
Use of systematic epidemiological methods in outbreak investigations from India, 2008-2016: A systematic review
}

\author{
Karishma K. Kurup ${ }^{\mathrm{a}, 1}$, Denny John ${ }^{\mathrm{b}, \mathrm{c}, 1}$, Manickam Ponnaiah ${ }^{\mathrm{a}, *, 1}$, Tijo George $\mathrm{e}^{\mathrm{a}, 1}$ \\ a ICMR School of Public Health, ICMR-National Institute of Epidemiology, Chennai, Tamil Nadu, India \\ ${ }^{\mathrm{b}}$ Campbell Collaboration, New Delhi, India \\ ${ }^{\mathrm{c}}$ ICMR-National Institute of Medical Statistics, New Delhi, India
}

\section{A R T I C L E I N F O}

\section{Keywords:}

Disease outbreaks

Outbreak investigations

Systematic reviews

India

\begin{abstract}
A B S T R A C T
Background: In the absence of a comprehensive review, we conducted a systematic review on the use of systematic approach in outbreak investigation using reports from India.

Objectives: The primary objective was to estimate the proportion of outbreak reports from India during 2008-16, that reported the steps in outbreak investigation. The secondary objectives were to (1) describe the outbreak reports by selected characteristics (source, investigating agency, disease, time, place and person) (2) estimate the proportion of outbreaks that conducted analytical and additional studies.

Methods: We searched eight electronic databases and grey literature for outbreak investigation reports among humans at community settings from India during 2008-2016. We developed a check-list based on the 10-steps approach used by Field Epidemiology Training Programme (FETP) of ICMR-National Institute of Epidemiology (ICMR-NIE), Chennai, India. This checklist was used to independently screen and extract data on general characteristics of the outbreak investigation reports and the steps completed. We adopted The Joanna Briggs Institute (JBI) check list for prevalence studies to examine the credibility and consistency. The protocol was registered at Prospero (CRD42017065038). We calculated proportion of reports that followed the steps in their investigation and descriptive statistics on selected characteristics.

Results: Of 10,657 articles screened, 136 articles were included for the review. Completion of the ten steps in the outbreak investigations was seen in $16 \%$ of reports. The highest level of completion was for drawing conclusion in outbreak investigation (98\%) and the lowest completion (29\%) was for developing a case definition by time, place and person followed by conducting an analytic study (24\%).

Conclusions: Outbreak reports from India either lacked application of systematic steps for investigation or failed to report the actual procedures followed. We recommend improving systematic investigation of outbreaks through training and supervision of outbreak response teams and encouraging publications.
\end{abstract}

\section{Introduction}

Outbreaks are public health emergencies that require a systematic investigation for early identification of causative factors so as to reduce morbidity, fatality and develop effective public health measures. ${ }^{1}$ While conducting an outbreak investigation one requires following a methodological approach. The United States of America Centers for Disease Control and Prevention (CDC), Atlanta proposes a 10-steps process in outbreak investigation that is adaptable to circumstances, resources available or suspected cause. ${ }^{1-3}$ The application of such a systematic approach allows in defining the outbreak, identifying the risk factors and application of interventions, that could alter the risk of transmission post-assessment. There exists a logical sequence in the 10steps followed during an outbreak investigation but in some situations, these steps may occur simultaneously. ${ }^{4}$

The use of such a systematic approach for conducting outbreak investigations is evident in solving many outbreak investigations of unknown etiology of commonly reported conditions. This is elicited by the systematic approach that was used in the investigation of hepatitis A caused by the consumption of raw shellfish in Pascagoula, Mississippi in 1968. ${ }^{5}$ Further, such systematic investigation of HIV pandemic resulted in identification of the risk factors well-before the confirmation of causative agent. In the Indian context, the utility of conducting a systematic outbreak investigation was demonstrated by the identification

\footnotetext{
* Corresponding author.

E-mail address: manickam@nie.gov.in (M. Ponnaiah).

${ }^{1}$ Authors have contributed equally.
} 
of the possible causative agent for recurrent outbreaks of a mystery illness in Muzaffarpur, Bihar that was first investigated in 2013 and later reinvestigated in $2014 .^{6-8}$

In India, the National Centre for Disease Control (NCDC) is the apex institution that deals with infectious disease research, surveillance, investigations and response in the country. NCDC is responsible for implementing India's Integrated Disease Surveillance Programme (IDSP) and mandates weekly reporting, investigation and response to outbreaks in different parts of the country. The NCDC recommends 10-step approach for outbreak investigations in India. ${ }^{1}$ Other public health institutions like medical colleges and public-private health agencies also conduct outbreak investigations in India.

Our initial review indicated that only a few of the outbreak investigations have focused on certain aspects like outbreak milestones. ${ }^{9}$ Few authors have identified the lack of methodology and failure to conduct analytical studies in outbreak investigations in India. ${ }^{10}$ In the absence of a comprehensive review of status of systematic approach in outbreak investigations from India, we conducted a systematic review on the extent of use of systematic approach in the investigation of disease outbreaks using studies and reports from India. The primary objective was to estimate the proportion of outbreak reports from India reported during 2008-16, that has systematically followed 10-steps in outbreak investigation. The secondary objectives were to describe these outbreak reports by selected characteristics (such as source, investigating agency, disease/health problem, time, place and person) and estimate the proportion of outbreaks that have conducted analytical studies and additional studies such as environmental investigations.

\section{Materials \& methods}

\subsection{Research question and review protocol}

The research question for this review is 'What is the extent of use of 10-steps approach in the disease outbreaks investigated reported from India during 2008-16'? The definition of disease outbreaks in this study is, "the occurrence of cases of an illness or specific health-related behavior or other health event, clearly in excess of normal expectancy in a community in a specific time period". ${ }^{11}$ A research protocol with details regarding the research question, search strategy, selection criteria, data extraction, primary and secondary outcomes and analysis plan was reviewed and approved by the Institutional Human Ethics Committee (IHEC) of Indian Council of Medical Research (ICMR)-National Institute of Epidemiology (NIE) [ICMR-NIE], Chennai and subsequently registered in the PROSPERO database (CRD42017065038) prior to the commencement of the review.

\subsection{Search strategy}

All outbreak investigation reports that were conducted in India during 2008-2016 were retrieved from published and grey literature. Grey literature refers to materials that are not published in peer reviewed journal. Search algorithm was prepared after reviewing the title, keywords and abstract of three studies and a study protocol related to systematic reviews previously done using outbreaks ${ }^{12-15}$

Search terms used in the strategy included "outbreak", "disease outbreak", "disease", "illness", "evaluation studies", "outbreak investigation", "epidemic", "cluster". The search was conducted from 12 May to 19 July 2017 and was limited to human studies. The databases searched were "Proquest", "Ovid", "Pubmed", "Cochrane Central", "ICMR-NIE's institutional reports ", " Indmed ", " Google Scholar "," Disease Volume Reports of IDSP ”, CDC Morbidity and Mortality Weekly Report", "Trip Database" (basic version with free access).

Search algorithm used in Pubmed was:(("disease outbreaks"[MeSH Terms] OR ("disease"[All Fields] AND "outbreaks"[All Fields]) OR "disease outbreaks"[All Fields] OR "outbreak"[All Fields]) AND ("evaluation studies as topic"[MeSH Terms] OR ("evaluation"[All Fields] AND "studies"[All Fields] AND "topic"[All Fields]) OR "evaluation studies as topic"[All Fields] OR "investigation"[All Fields])) AND India[Title/Abstract] AND ("2008/01/01"[PDAT]: "2016/12/ 31"[PDAT]). Search algorithms to retrieve articles from all identified databases are reported in (S1 Table).

\subsection{Selection criteria}

All retrieved articles were initially uploaded into the Covidence software (Julian Elliot, Australia). We identified and removed duplicate studies (defined as those similar looking studies identified from different databases during the search and not that of multiple reports of a single outbreak reported in multiple studies). Two independent reviewers screened the articles as per the inclusion and exclusion criteria. The title and abstract of all the articles uploaded into Covidence were initially screened and relevant full-text articles were obtained. All outbreak investigation reports (published articles and reports) from 2008 to 2016 conducted in community settings in India were included irrespective of the presence of an analytic investigation in the report. The year 2008 was chosen since the IDSP began weekly reporting of outbreaks from November 2007 onwards. Studies were limited to human participants.

\subsection{Data extraction and analysis}

An extraction form was prepared and was pilot-tested using fiveoutbreak investigation reports. All disagreements were discussed with the four members of the review team and a consensus was reached for any further modifications. Two reviewers independently extracted the data from full-text articles using the form (S2 Table). The extracted details include type of articles by publication status, nature of infection, disease by ICD 10 classification, agency that conducted the investigation (government or others; government agency included NCDC, ICMR, Government-run medical colleges and army institutions; while others were non-governmental organizations). We categorized the number of agencies involved in the investigation as single agency or multiple agencies. "Single agency" referred to investigations conducted by only one agency and "multiple agencies" referred to investigations conducted by more than one agency, for e.g., ICMR partnered/conducted the outbreak investigation with another agency or agencies.

Further, we abstracted information on year of outbreak and publication, place and location in the State and person characteristics (availability of population figures for outbreak area/setting, case attack rates, by gender, by age group, and case fatality). On the steps used, we collected details on each of the individual steps, type of analytical study and environmental investigations conducted. The completion of steps in outbreak investigation was described as cumulative proportion and for that of each step of the outbreak investigation and subcomponents of each of those steps. The gaps in reporting at each level were identified and summarized. For describing each step, we adapted the outbreak investigation checklist for 10-steps approach used by the Field Epidemiology Training Programme (FETP) at ICMR-NIE. ${ }^{1}$ (S4 Table) The 10-steps were classified into the main steps and their subcomponents. Reporting of the main steps and subcomponents of those steps were taken as completion of the steps, and the completion of any one subcomponent of the step was taken as completion of the main step. The preferred reporting items for systematic review and meta-analysis (PRISMA) guidelines were followed in reporting the methods and results of the review (S3 Table PRISMA guidelines for reporting systematic reviews).

\subsection{Risk of bias assessment}

Currently, there is no recognized technical tool for quality appraisal tool of outbreak investigation field reports. A critical appraisal tool for 
judging bias was developed by modifying the The Joanna Briggs Institute (JBI) checklist for prevalence studies with criteria relevant for reporting an outbreak investigation. ${ }^{16} \mathrm{~A}$ score of one was assigned to each component and 0.5 to each subcomponent of the checklist. A higher weightage was given to criteria $1,2.7$ and 8 this was decided based by review team members (S5 Table). Credibility was judged by the completion of the four major criteria and consistency was judged by the adherence in reporting an outbreak by time, place and person with relevant analysis and recommendations. Quality of study was categorized into low quality (If even one of the four criteria was not covered) incomplete descriptive findings and analytic findings), medium quality (All the four criteria were covered) and high quality (for a score of 6-7).

\section{Results}

\subsection{Screening of articles}

From the initial search conducted 10,657 articles were identified, excluding duplicates, 6014 articles were screened by title and abstract. From these 393 articles were screened for full-text and 137 articles were included in the final analysis. The full-text could not be obtained for one study, and hence, a total of 136 reports were included for final analysis (Fig. 1).

\subsection{General characteristics of outbreak investigation reports}

Majority of the outbreak investigation reports are from peer-reviewed journals $(n=114,84 \%)$. Of the total reports $(n=136), 22$ were from the IDSP's surveillance reports. Majority of the reports were from investigation by government agencies $(n=130,96 \%)$. Of the total reports, ICMR $(\mathrm{n}=57 ; 42 \%)$ followed by academic institutions ( $\mathrm{n}=52 ; 38 \%)$ and IDSP $(\mathrm{n}=29 ; 22 \%)$ were the major investigating agencies involved. Twenty-one outbreak reports (15\%) were contributed by FETP at ICMR-NIE. Almost half of the reviewed reports involved investigation by multiple agencies (Fig. 2).

Outbreak reports published during 2008-16 increased by $60 \%$ and with the maximum number of reports appearing in 2016 (14.7\%, $\mathrm{n}=20$ ) (Fig. 3) The published outbreaks had occurred during 2002-2016 with maximum $(\mathrm{n}=20)$ in 2007.

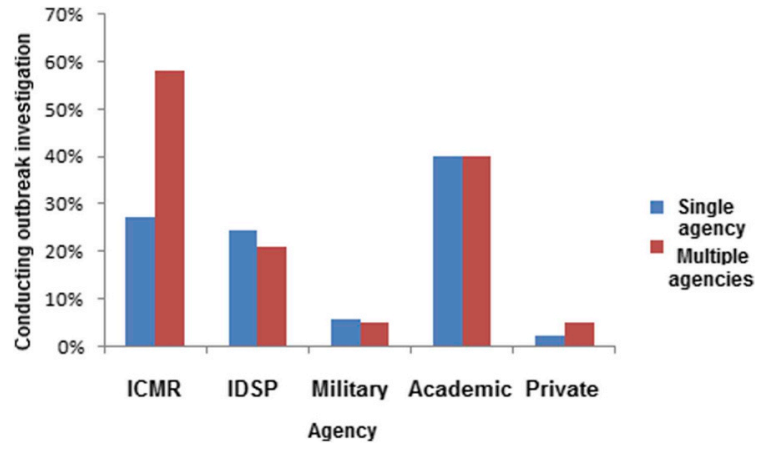

Fig. 2. Frequency of outbreak investigations conducted by single and multiple agencies.

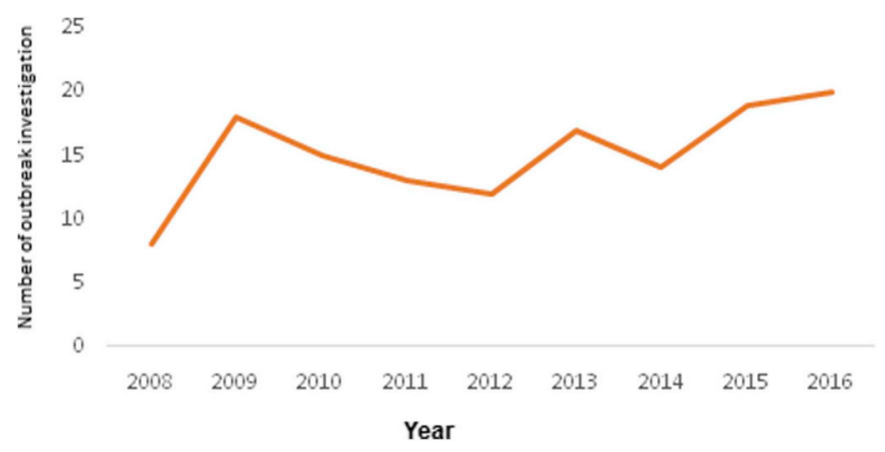

Fig. 3. Reporting of outbreak investigations by the year published in India, 2008-2016.

Maximum number of outbreak reports came from West Bengal $(\mathrm{n}=14)$, Odisha $(\mathrm{n}=11)$ and Madhya Pradesh $(\mathrm{n}=10)$, in two outbreak investigation reports at military settings and the location was limited to South India. Outbreaks from West Bengal $(n=7)$ and Odisha $(n=5)$ were due to intestinal infections, whereas Madhya Pradesh reports were that of intestinal $(n=3)$ or arthropod-borne viral diseases $(n=3)$.

Of the total reports, $102(75 \%)$ mentioned the population of the
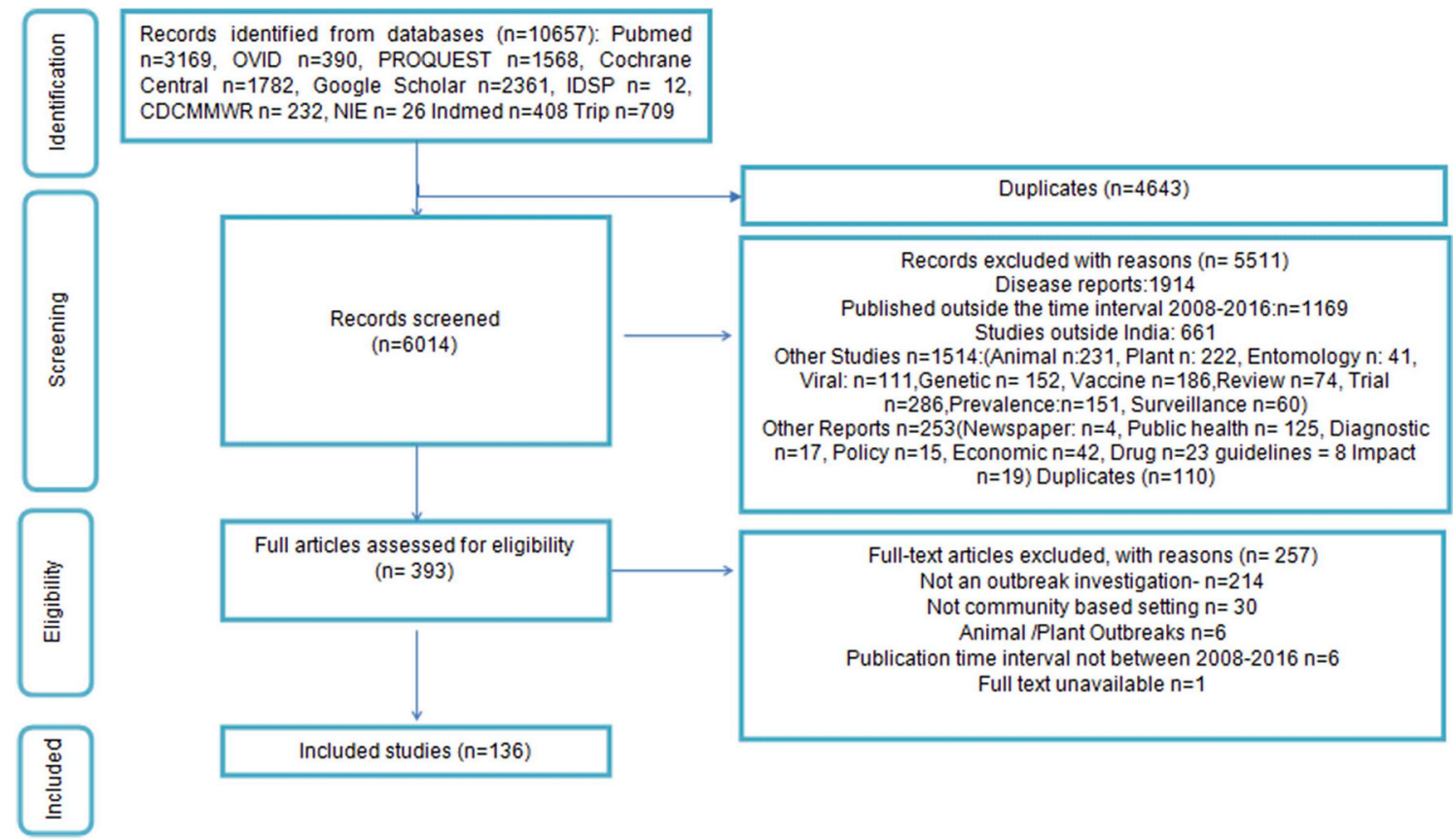

Full-text articles excluded, with reasons $(n=257)$

Not an outbreak investigation- $n=214$

Not community based setting $n=30$ Animal /Plant Outbreaks $n=6$

Publication time interval not between $2008-2016 n=6$ Full text unavailable $n=1$

Fig. 1. PRISMA flowchart showing the screening process, Outbreak Investigation reports in India from 2008 to 2016 were screened using this process. 
Table 1

Distribution of outbreak reports by disease using ICD 10 classification.

\begin{tabular}{|c|c|c|}
\hline ICD 10 classification & Diseases in each classification & Outbreak reports \\
\hline Intestinal infectious disease & Acute diarrheal disease, Cholera, Gastroenteritis, Shigellosis, Typhoid & 33 \\
\hline Other bacterial disease & Diphtheria, Meningeal, Pertussis & 8 \\
\hline Certain zoonotic bacterial disease & Plague, Anthrax, Leptospirosis & 7 \\
\hline Rickettsiosis & Scrub typhus & 3 \\
\hline Arthropod-borne viral disease & $\begin{array}{l}\text { West Nile, Kyanasur Forest Disease, Dengue, Crimean Congo Hemorrhagic Fever, Chandipura, } \\
\text { Chikungunya }\end{array}$ & 26 \\
\hline $\begin{array}{l}\text { Viral infections characterized by skin \& mucous membrane } \\
\text { lesions }\end{array}$ & Buffalopox virus, chicken Pox, Hand Foot and Mouth disease, Rubella, Measles & 20 \\
\hline Viral hepatitis & Hepatitis A, E, B & 19 \\
\hline Protozoal disease & Malaria & 5 \\
\hline Toxins & Photokeratoconjunctivitis, Dropsy, Food Poisoning & 5 \\
\hline Helminths & Filariasis, Trichinella & 2 \\
\hline Other viral disease & Japanese Encephalitis, Nipah & 3 \\
\hline Pneumonia \& Influenza & Influenza $\mathrm{A}, \mathrm{B}$, Influenza like illness, Pneumonia & 5 \\
\hline
\end{tabular}

outbreak setting. Little over half of the reports $(n=69)$ specified the attack rate and in 36 of them case fatality was described. Further age $(n=42)$ and gender $(n=44)$ specific attack rates were available in one-third of the reviewed reports.

Most of the diseases reported (96\%) in outbreak reports were infectious nature. Most common among the disease outbreaks with reference to ICD-10 classification were intestinal infectious disease $(\mathrm{n}=33)$, arthropod-borne viral disease $(\mathrm{n}=26)$ and viral infections characterized by skin $\&$ mucous membrane lesions $(n=20)$ (Table 1$)$.

Of the 134 reports belonging to a known etiology or pathogen, 92 needed extensive work-up. While two reports of unknown etiology/ unknown pathogen needed extensive work-up.

\subsection{Outbreak investigation reports completing the steps of outbreak investigation and following a systematic approach}

Twenty-two of the 136 reports (16\%) completed all the steps in outbreak investigation. Majority of such reports involved investigation by multiple agencies $(\mathrm{n}=15)$ (Table 2 ).

Our analysis suggests that as agencies leading the investigations, eight of the 15 reports from the non-governmental, 20 of 130 reports from governmental and four of 63 reports from academic institutions used 10-step approach. In terms of investigating agencies, 14 reports from ICMR, 13 from FETP at ICMR-NIE and there were four reports each from IDSP and academic institutions had all the 10-steps followed (Fig. 4).

\subsection{Completion of subcomponents for each step in the outbreak reports}

All the outbreak reports reported the first step of the outbreak investigation but when reviewed by subcomponents there was a major shortfall in reporting of artifacts by reporting any changes in surveillance or population. In the second step of outbreak investigation, a clinical description was available in all the outbreak reports. However, there was failure to report the mechanism of safe packaging and transport of specimens in many outbreak reports. Apart from the routine investigations correlating with the clinical suspicion, histo-

Table 2

Completion of steps of outbreak investigation by single and multiple agencies.

\begin{tabular}{lll}
\hline Total steps completed & Reports by single agency & Reports by multiple agencies \\
\hline 4 & 2 & 1 \\
5 & 4 & 3 \\
6 & 5 & 7 \\
7 & 15 & 11 \\
8 & 27 & 23 \\
9 & 10 & 6 \\
10 & 5 & 15
\end{tabular}

pathological samples were also collected from animals and humans to confirm the diagnosis. This was specifically seen in zoonotic outbreaks like buffalo pox $(n=3)$ and anthrax $(n=4)$. Defining a case, which is the third step, was reported in most of the outbreak reports. However, time, place and person components in a case definition were mentioned in one-third reports only $(\mathrm{n}=40)$. Generating a hypothesis forms step five of an outbreak investigation and it is objectively based on the distribution by time, place and person. The description by time through an epidemic curve and "population-based incidence by age and gender" completed in $62 \%(n=84)$ and $90 \%(n=123)$ reports respectively. However, using "spot map to describe the outbreak by place" "conducting hypothesis-generating interviews among case-patients" were mentioned and completed in $32 \%(n=43)$ and $69 \%$ of reports respectively. Step six of testing of the hypothesis generated, was completed in 33 of the 136 (24\%) reports with majority using case-control design $(n=23)$ followed by retrospective cohort study $(n=9)$ and spatial analysis $(\mathrm{n}=1)$. Once hypothesis is established "Comparison of hypothesis with established facts" was seen in most of the outbreak reports, however, the discussion between colleagues, peers and supervisors was reported only in $36 \%$ of reports(Table 3 ).

\subsection{Quality of the outbreak reports}

Overall, 58 reports (45\%) were of high quality, 22 (16\%) were of medium quality, and $56(41 \%)$ reports were of low quality. Since $40 \%$ $(n=55)$ of the included studies were conducted by ICMR either directly or in partnership with other agencies, we analyzed their quality of outbreak reports separately. Of the 55 reports published by ICMR, 23 were of high quality, 10 were of medium quality and 22 were of low quality.(S6 Table)

\section{Discussion}

We did a systematic review of use of classical steps of outbreak investigation in human population in community settings in India from published reports during 2008-16. Our findings suggest that low frequency of the reviewed reports had used the 10-steps of outbreak investigation and maximum outbreaks were investigated by government agencies.

Outbreak investigation is a major public health responsibility of every government. ${ }^{1,2}$ Poor surveillance and response can result in disastrous consequences. The plague outbreak in Surat, 1994, is one such example that led to a near international isolation of India. ${ }^{17}$ This outbreak led to initiatives and policy decisions to develop a robust surveillance system to prevent such future outbreaks. ${ }^{2}$ In fact, India established the National Surveillance Programme for Communicable Diseases in 1997 and was later on up-scaled to IDSP in 2004 with the assistance from World Bank. According to a recent review of IDSP 


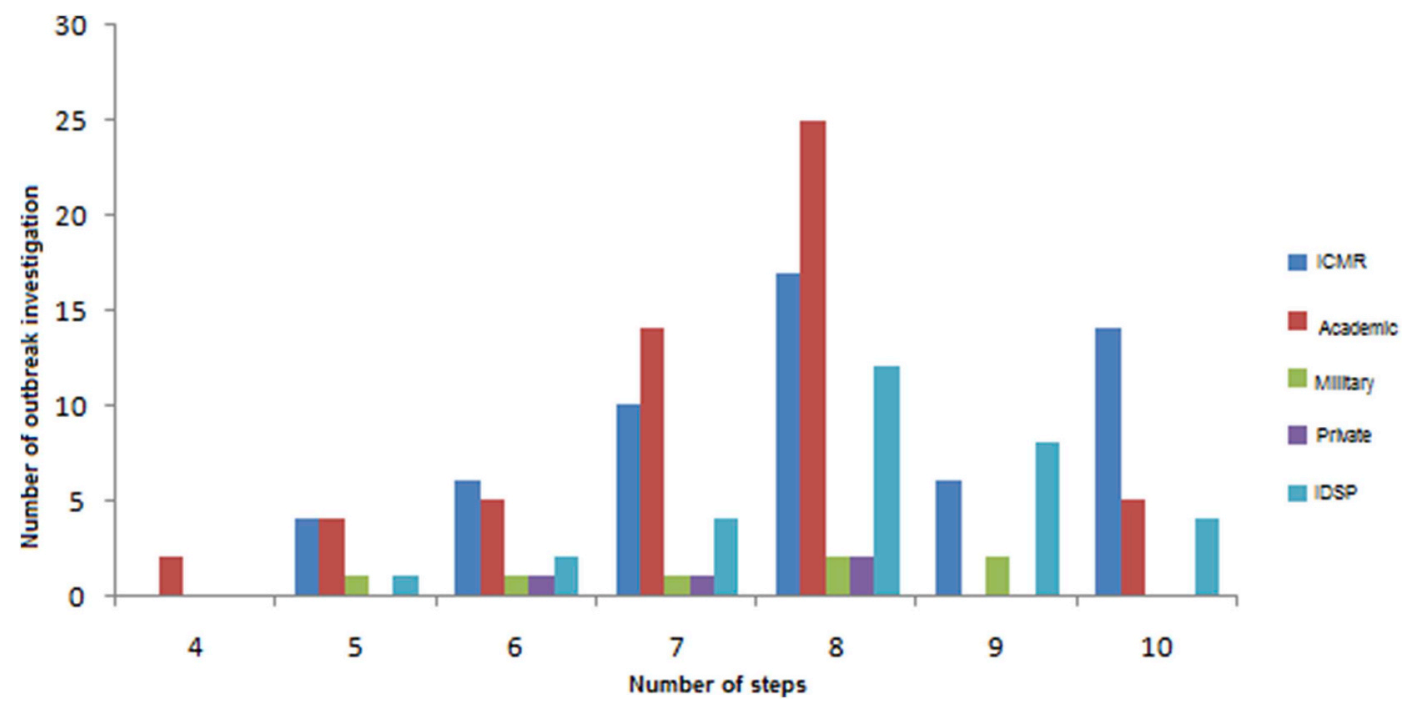

Fig. 4. Completion of steps by the type of agency conducting outbreak investigation.

(2015), the issues still needing attention were that of poor quality surveillance and reporting, limited specimen testing in outbreaks and low laboratory standards ${ }^{18}$; lack of useful data and data analytics; nonfunctional surveillance committees, a higher priority to disease of other vertical programmes; no defined core competencies for staff, high vacancies, contractual post and lacunae in the fund release ${ }^{18}$ We presume that combination of such identified issues could have contributed to the gaps that were observed in the systematic approach to outbreak investigations in India. ${ }^{6-8,10}$

Our findings of use of 10-step approach in majority of the outbreaks reported by FETP or ICMR led investigations is pointer to the fact that collaboration among public health agencies and quality trained graduates bring to the table use of methodological approach to outbreak investigation and effective containment. Such evidence from India is worth noting for policy makers, whereas, such international experience had earlier been documented in case of Severe Acute Respiratory Syndrome in China ${ }^{19}$ and Canada 2003, Avian influenza in Thailand 2004, Ebola outbreak in Uganda in 2000. ,9 $^{2,9}$
ICMR is the apex body for formulating, coordinating and promoting research. ${ }^{20}$ Most of the reviewed outbreak investigation reports were handled by ICMR either alone or in partnership. ${ }^{10}$ However, even with the availability of quality training, a good surveillance system, and adequate resources, our study documents that there are reporting gaps in the systematic approach to outbreak investigation. Previous studies recognize lack of reporting of the actual procedures followed in outbreak investigation by the investigating teams. ${ }^{10,12,24}$ In order to improve better reporting practices of outbreak investigations in published literature, a 22 item checklist - "Outbreak Response and Intervention studies of Nosocomial Infection" (ORION) was developed in 2007 by Stone SP et al. ${ }^{21} \mathrm{~A}$ follow-up review close to a decade after the ORION guidelines were framed, reported a failure in the completion of reports as per these guidelines. ${ }^{22}$ Our study has followed the 10- steps approach since both international and national agencies have advocated the need to stringently follow these ten steps while conducting an outbreak investigation. Nevertheless, it is possible that the authors might have overlooked reporting of these steps based on the journal's target

Table 3

Distribution of outbreak reports (2008-16) by the completion of subcomponents for each step in an outbreak investigation, India.

\begin{tabular}{|c|c|c|}
\hline $\begin{array}{l}\text { Steps in an } \\
\text { Outbreak Investigation }\end{array}$ & Components & Reports showing completion \% (N) \\
\hline \multirow{4}{*}{ Step 1: Determine existence of an outbreak } & Excess of cases & $100 \%(136)$ \\
\hline & Checking for the background rates of disease & $45 \%(61)$ \\
\hline & Changes in surveillance system & $9 \%(12)$ \\
\hline & Changes in the population & $9 \%(12)$ \\
\hline \multirow[t]{3}{*}{ Step 2:Confirming the diagnosis } & Clinical description of a few cases to raise hypothesis in terms of diagnosis & $97 \%(132)$ \\
\hline & Collecting the right biologic specimens to confirm the suspected diagnosis & $95 \%(129)$ \\
\hline & Safe transport and packaging of biological samples in right laboratory & $25 \%(34)$ \\
\hline Step 3: Define a case & Case definition mentioned & $75 \%(102)$ \\
\hline \multirow[t]{2}{*}{ Step 4: Search for cases } & Search for cases & $86 \%(117)$ \\
\hline & Line-listing of cases & $26 \%(35)$ \\
\hline \multirow[t]{4}{*}{ Step 5: Generate hypothesis using descriptive findings } & Description of the outbreak by time through an epidemic curve & $62 \%(84)$ \\
\hline & Spot map to describe the outbreak by place & $32 \%(43)$ \\
\hline & Population-based incidence by age and gender & $90 \%(123)$ \\
\hline & Conducting hypothesis-generating interviews among case-patients & $69 \%(94)$ \\
\hline Step 6:Test hypothesis with analytical study & Conducting an analytic study & $24 \%(33)$ \\
\hline \multirow[t]{2}{*}{ Step 7: Draw conclusions } & Analysis of the analytic study & $23 \%(31)$ \\
\hline & Formulate conclusions that explain facts & $99 \%(134)$ \\
\hline \multirow{3}{*}{ Step 8: Compare hypothesis with established facts } & Conducting an environmental/additional investigation to confirm hypothesis & $72 \%(98)$ \\
\hline & Review of literature & $85 \%(116)$ \\
\hline & Discuss conclusions with colleagues, peers and supervisors & $36 \%(49)$ \\
\hline Step 9: Communication of findings & Communication of findings & $65 \%(88)$ \\
\hline \multirow[t]{2}{*}{ Step 10: Execute preventive measures } & Formulating clear specific recommendations & $93 \%(126)$ \\
\hline & Relevance and effectiveness of recommendation with implementation & $82 \%(112)$ \\
\hline
\end{tabular}


audience and word count limitations of the journal. However, by using this review tool, we would also like to draw attention to the need for guidelines while reporting such field-based investigations. Hence, only through mandatory compliance with guidelines while publishing an outbreak investigation, we can improve the quality of reporting of available information. Reporting the steps holds importance in ensuring that a systematic methodology and transparency are followed and clarifies any concerns raised by the audience in the conduct of an outbreak investigation.

The significance of these steps is that it contributes to early detection and developing effective measures that addresses the population exposed, the causality criteria and sources identified from an analytic study. ${ }^{1,6}$ One may argue that often during emergency response, steps are missed/overlooked leading to "quick and dirty investigations". However, uniformity of data quality and timeliness of outbreak reporting system are key considerations for data sharing between countries. $^{23}$

The strength of this review is that we examined the methodological completion of individual steps and its subcomponents of 10-step outbreak investigation checklist followed by FETP that is universally known for promoting hands-on training on the use of such systematic approach. Hence, we could identify the essential steps that were least reported. The lack of information in preparing case definitions and conducting analytic studies have been reported in previous studies $^{6,7,12,24,25}$ and supportive evidence was also available from the present study. Further, on the basis of our review, we highlight the importance of specimen collection and histo-pathological examination and the conduct of autopsies as and when indicated.

Our review has certain limitations. We relied on published sources, that could have led us to under or over-estimate the use of 10-steps in the investigated reports. We could have under-estimated the actual use of systematic outbreak investigation methodology in the field. However, actual use may be difficult to arrive at and hence, we did consult grey literature to partially overcome this limitation. On the other hand, we could have over-estimated the use of completion of 10steps in the published outbreaks due to publication bias associated with such investigations. This limitation is difficult to avoid and our estimates are available by investigating agencies that allows us to interpret accordingly. It may be possible that public health trainees (such as FETP) may tend to accurately report the systematic methodology due to the academic rigor and expectations. However, our primary interest was in estimating proportion of published outbreak reports following 10-steps irrespective of the author profile. Despite these limitations, our findings do provide important information on existing gaps that require immediate attention from surveillance and outbreak response authorities, investigating agencies and may be publishing houses. ${ }^{18}$ Additionally, we also conducted critical appraisal of the study designs using a modified JBI checklist to estimate the quality of the reporting.

\section{Conclusion}

This review reports existing gaps in the use of stepwise systematic approach in outbreak investigation, which is an international norm and rightly is incorporated in the Indian guidelines. The gap in completion of all the steps of outbreak investigation could be due to failure to either apply systematic steps during investigation or report the actual procedures followed. We recommend improving scientific investigation of outbreaks in India by strengthening quality of training, supervision of outbreak response, provision of resources, developing mandatory reporting guidelines and encouragement to publish.

\section{Acknowledgements}

We acknowledge the support of the Dr. Manoj Murhekar, Director of
ICMR-NIE and India FETP at ICMR-NIE to conduct the review; Dr. Tarun B, India FETP coordinator and Dr. P Ganeshkumar, Faculty, India FETP, ICMR-NIE who provided valuable comments for the review. Mr. S. Satish, Librarian, ICMR-NIE for the support. The training provided by The Campbell Collaboration during its July 2017 workshop at New Delhi is acknowledged.

\section{Appendix A. Supplementary data}

Supplementary data to this article can be found online at https:// doi.org/10.1016/j.cegh.2019.02.010.

\section{References}

1. Murhekar M, Moolenaar R, Hutin Y, Broome C. Investigating outbreaks: practical guidance in the Indian scenario. Natl Med J India. 2008 Dec 1;22:252-256.

2. Principles of Epidemiology: Home|Self-Study Course SS1978|CDC [Internet]. Cdc.gov. 2017 [cited 28 October 2017]. . Available from: https://www.cdc.gov/ ophss/csels/dsepd/ss1978/index.html.

3. Goodman RA, Buehler JW, Koplan JP. The epidemiologic field investigation: science and judgment in public health practice. Am J Epidemiol. 1990 Jul;132(1):9-16.

4. Reingold A. Outbreak investigation-a perspective. Epidemiol Bull. 2000 Jun;21(2):1-7

5. Brachman PS, Thacker SB, Centers for Disease Control and Prevention (CDC). Evolution of epidemic investigations and field epidemiology during the MMWR era at CDC-1961-2011. MMWR Suppl. 2011 Oct 7;60(4):22-26.

6. Jacob John T, Das M. Acute encephalitis syndrome in children in Muzaffarpur: hypothesis of toxic origin. Curr Sci. 2014 May 1;106:1184-1185.

7. Shah A, Jacob John T. Recurrent outbreaks of hypoglycaemic encephalopathy in Muzaffarpur, Bihar. Curr Sci. 2014 Aug 25;107(4):1184-1185.

8. Shrivastava A, Srikantiah P, Kumar A, et al. Outbreaks of unexplained neurologic illness - Muzaffarpur, India, 2013-2014. MMWR Morb Mortal Wkly Rep. 2015 Jan 30;64(3):49-53.

9. Bruckner C, Checchi F. Detection of infectious disease outbreaks in twenty-two fragile states, 2000-2010: a systematic review. Conflict Health. 2011 Aug 23;5:13.

10. Balraj V. Investigation of outbreaks in India. How good are we at it? Indian Pediatr. 2003 Oct; 40(10):933-938.

11. N I C D. List for Central Rapid Response Team for Disease Outbreak Investigation Including Avian Influenza [Internet]. Government Of India, National Institute Of Communicable Diseases; 2008. Available from: http://www.nicd.nic.in/checklist. pdf.

12. Nguyen Tien Huy, Le Khac Linh, Hoang Thi Nam Giang, et al. Deaths of emerging and re-emerging infectious diseases outbreaks, epidemics, and pandemics in the last 10 years: a systematic review [Internet]. [cited 2017 Oct 6]. . Available from: http:// www.crd.york.ac.uk/PROSPERO/display record.asp?ID = CRD42016038138.

13. Smith CM, Comber SCL, Fry H, Bull M, Leach S, Hayward AC. Spatial methods for infectious disease outbreak investigations: systematic literature review. Euro Surveill. 2015 Oct 1;20(39):30026.

14. Kanungo S, Sah BK, Lopez AL, et al. Cholera in India: an analysis of reports, 1997 2006. Bull World Health Organ. 2010 Mar;88(3):185-191.

15. Dikid T, Jain SK, Sharma A, Kumar A, Narain JP. Emerging \& re-emerging infections in India: an overview. Indian J Med Res. 2013 Jul;138(1):19-31.

16. The Joanna Briggs Institute. Critical Appraisal Tools - JBI [Internet]. . Available from: http://joannabriggs.org/research/critical-appraisal-tools.html.

17. Deodhar NS, Yemul VL, Banerjee K. Plague that never was: a review of the alleged plague outbreaks in India in 1994. J Public Health Policy. 1998;19(2):184-199.

18. WHO. Joint Monitoring Mission Report Integrated Disease Surveillance Programme. 2015; 2015 .

19. Nsubuga $\mathrm{P}$, White ME, Thacker SB, et al. Public health surveillance: a tool for targeting and monitoring interventions. In: Jamison DT, Breman JG, Measham AR, eds Disease Control Priorities in Developing Countries [Internet]. second ed. Washington (DC): World Bank; 2006.

20. Kant L. Combating emerging infectious diseases in India: orchestrating a symphony. $J$ Biosci. 2008 Nov;33(4):425-427.

21. Stone SP, Cooper BS, Kibbler CC, et al. The ORION statement: guidelines for transparent reporting of outbreak reports and intervention studies of nosocomial infection. Lancet Infect Dis. 2007 Apr;7(4):282-288.

22. Wieland K, Chhatwal P, Vonberg R-P. Outbreak reporting a decade after ORION: where do we stand? Lancet Infect Dis. 2017 May 1;17(5):476.

23. Lawpoolsri S, Kaewkungwal J, Khamsiriwatchara A, et al. Data quality and timeliness of outbreak reporting system among countries in Greater Mekong subregion: challenges for international data sharing. [cited 2019 Jan 18]. PLoS Negl Trop Dis [Internet]. 2018 Apr 25;12(4). Available from: https://www.ncbi.nlm.nih.gov/pmc/ articles/PMC5937798/.

24. Pons W, Young I, Truong J, et al. A systematic review of waterborne disease outbreaks associated with small non-community drinking water systems in Canada and the United States. PLoS One. 2015 Oct 29;10(10):e0141646.

25. Dasari V, Kaur P, Murhekar MV. Rickettsial disease outbreaks in India: a review. Ann Trop Med Public Health. 2014 Nov 1;7(6):249. 